\title{
Spin-wave Brillouin Scattering in CoPt Alloy Films with Perpendicular c-axis Orientation of hcp-Co
}

\section{Akihiro MURAYAMA ${ }^{1}$, Masao MIYAMURA ${ }^{1}$, Kazushige NISHIYAMA ${ }^{2}$, and Yasuo OKA ${ }^{2}$}

1) R\&D Div., ASAHI-KOMAG Co.Ltd., Hachimannhara, Yonezawa 992-11, Japan

2) Research Institute for Scientific Measurements, TOHOKU University, Sendai 980, Japan

Surface spin waves in CoPt alloy films with perpendicular c-axis orientation of hcp-Co have been studied by Brillouin light scattering for the high-density magnetic recording. The spin-wave energy depends on the Pt-content, which is explained by the change of the perpendicular magnetic anisotropy, as well as the saturation magnetization. From the dependence of the spin-wave energy on the magnetic field, magnetic constants are derived, such as g-value and uniaxial anisotropy constant. Line broadening of the spin-wave Brillouin spectra are also discussed.

\section{INTRODUCTION}

A rapid increase in the recording density of magnetic thin film media requires fine control of the crystal structure of Co-based magnetic alloy films, such as crystal orientation, crystallinity, grain size and grain separation. Even in high-density longitudinal recording media, perpendicular magnetic anisotropy which can play a significant role in the media noise reduction[1] must be taken into account. However, a little work was reported for the micro-magnetic properties of such kind of the longitudinal magnetic films having a perpendicular anisotropy.

On the other hand, spin-wave Brillouin scattering is a powerful tool for the evaluation of the magnetic properties from the microscopic point of view[2]. Saturation magnetization $\left(M_{s}\right)$, g-value, exchange stiffness and magnetic anisotropies of thin films can be obtained, as well as the ferromagnetic resonance. In metallic films, the scattering due to a surface spin-wave called as DamonEshbach (DE) mode which propagates along the film surface is strongly observed, since the skin depth of the visible light in such metallic films is less than about 200$\dot{A}$. Therefore, it is useful for the magnetic evaluation in very thin magnetic films.

We have studied spin-wave Brillouin scattering in the $230 \dot{\mathrm{A}}$-thick CoPt alloy films by changing the Ptcontent up to 24at.\%. These films show a strong c-axis orientation of hcp-Co perpendicular to the film plane leading to a perpendicular anisotropy $\mathrm{K}_{\mathrm{u}}$. This anisotropy is superimposed by the in-plane shape anisotropy $2 \pi M_{s}{ }^{2}$. The effective magnetic anisotropy is given by,

$$
\mathrm{K}_{\text {ueff }}=2 \pi \mathrm{M}_{\mathrm{s}}{ }^{2}-\mathrm{K}_{\mathrm{u}} \quad---(1) \text {. }
$$

By this definition, $K_{\text {ueff }}>0$ means the resulting easy axis is in-plane. Results are compared with those in the film having a random orientation of the $c$-axis.

\section{EXPERIMENTAL PROCEDURE}

Two types of CoPt alloy films were fabricated on $\mathrm{Si}$ substrates by RF-sputtering using a Co target with $\mathrm{Pt}$ chips. Type I films had a random c-axis orientation of hcpCo. During the deposition of the Type I films, substrates were heated at $150^{\circ} \mathrm{C}$. Type II films which were grown under an external magnetic field of 60 Oe perpendicular to the substrate surface had a strong c-axis orientation perpendicular to the film plane. In the deposition, no intentional heating was introduced. The Pt content of the film was changed from 0 to 24 at. $\%$ by changing the number of the Pt chips. Actual compositions of the films were analyzed by EPMA. Magnetic hysteresis properties were measured by VSM. Film structure was evaluated by $\mathrm{X}$-ray diffraction, SEM and TEM.

Spin-wave Brillouin scattering was observed in the back scattering geometry by a tandem 5-pass FabryPerot interferometer. A $514.5 \mathrm{~nm}$ line of an Ar ion laser with a power of $100 \mathrm{~mW}$ was used as an excitation light source. Brillouin scattering from surface acoustic waves was removed by using a polarizer . External magnetic field up to $4 \mathrm{kOe}$ was applied parallel to the film plane. 


\section{RESULTS AND DISCUSSION}

In the Type II films, a strong c-axis orientation perpendicular to the film plane is observed at the $\mathrm{Pt}$ contents of 15 and 24at.\% by X-ray diffraction. Figure 1 shows X-ray diffractions from Type II- $\mathrm{Co}_{85} \mathrm{Pt}_{15}$ and Type I-Co ${ }_{86} \mathrm{Pt}_{14}$ alloy films. The Type II film shows a strong peak from (002) planes parallel to the substrate surface. In the Type I film, CoPt crystal grains with a random orientation of hcp-Co is observed by a combination of TEM and the transmission electron diffraction.
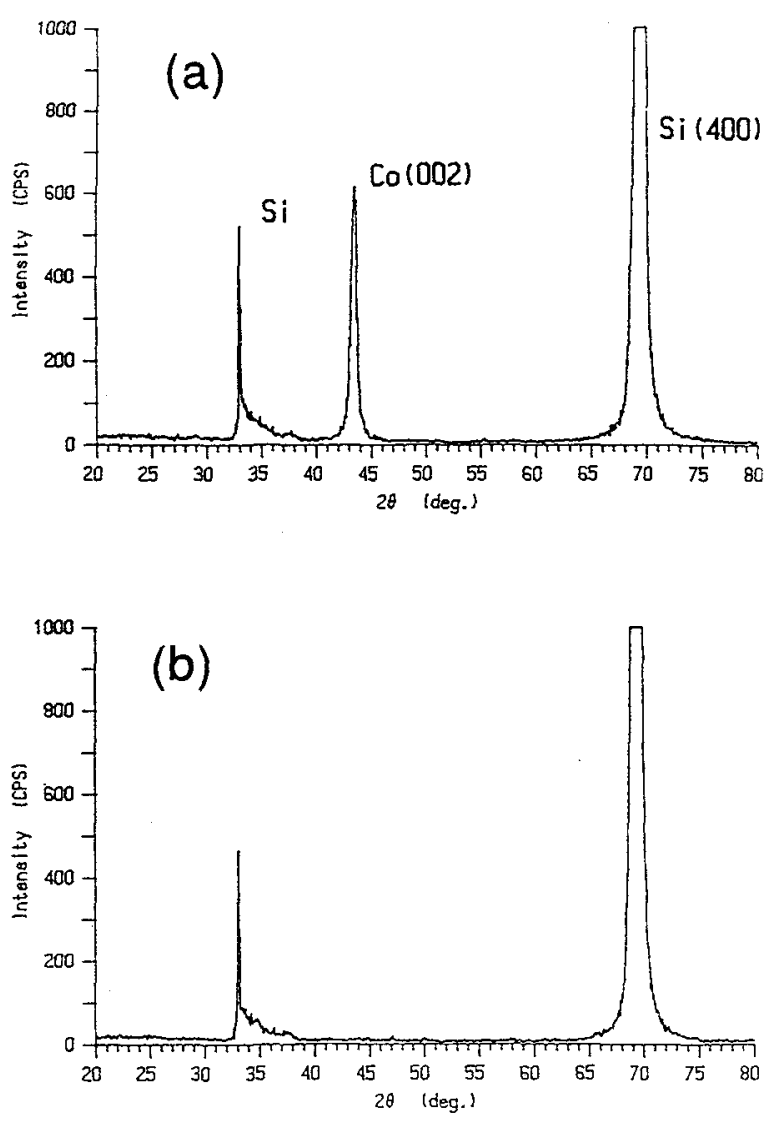

Fig.1 X-ray diffractions from Type II - $\mathrm{Co}_{85} P t_{15}$ (a) and Type I- $\mathrm{Co}_{86} \mathrm{Pt}_{14}$ (b) alloy films.

In-plane coercivity in the Type II films depends on the Pt-content. Moreover, Type II films at the Ptcontents of 15 and 24 at.\% show relatively square hysteresis loops by applying the magnetic field perpendicular to the film plane, which is considered to be related to the strong $c$-axis orientation perpendicular to the film plane.
Hysteresis curves by applying external fields in both directions of the film plane for the Type II $-\mathrm{Co}_{85} \mathrm{Pt}_{15}$ film are shown in Figure 2.

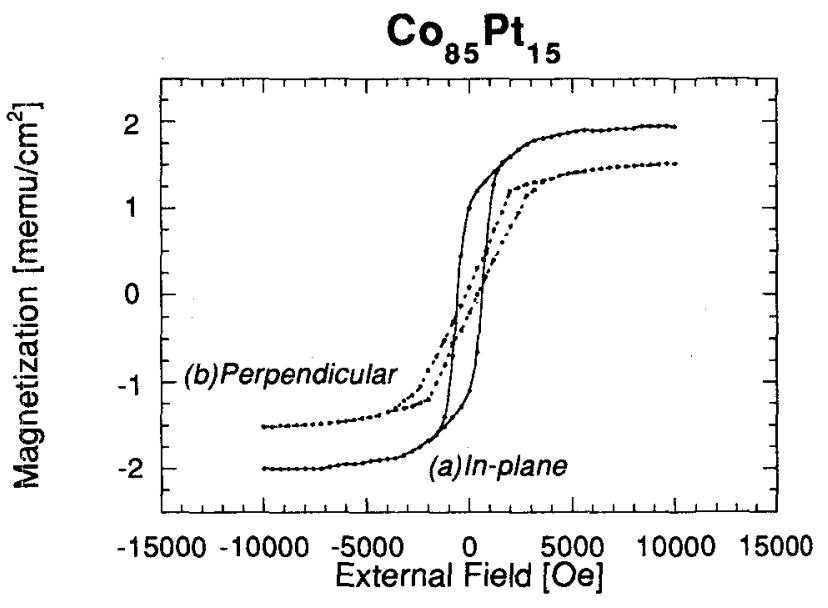

Fig.2 Hysteresis curves in Type II - $\mathrm{Co}_{85} \mathrm{Pt}_{15}$ film, with applying external fields parallel( $a$ ) and perpendicular(b) to the film plane.

Spin-wave Brillouin spectra in CoPt alloy films of Type-II at various $\mathrm{Pt}$ contents, under magnetic field of $2.4 \mathrm{kOe}$ parallel to the film plane, are shown in Figure 3. Strong peaks observed in the figure are assigned as the DE mode by the dependence of the spin-wave energy on the angle between incident light and the film surface. The spinwave energy is shown to be affected by the Pt-content. The DE spin-wave energy is derived from the Landau-Lifshits torque equation and Maxwell equations by considering surface boundary conditions[3]. Dutcher et.al. have treated the spin-wave energy of the uniform mode which is excited inside the film, in the Brillouin scattering from $\mathrm{Fe}$ superlattices grown on $\mathrm{Cu}$, with large uniaxial anisotropies perpendicular to the film plane[4]. In their analysis, spinwave energy was evaluated by considering the effective anisotropy field, as follows:

$$
\left(4 \pi D_{\perp} M_{s}\right)_{e f f}=4 \pi D_{\perp} M_{s}-2 K_{u} / M_{s},-- \text { (2) }
$$

where $K_{u}$ is the uniaxial anisotropy constant and $D_{\perp}$ is an effective demagnetizing factor. The same consideration can be made for the CoPt alloy films which has a strong perpendicular c-axis orientation. For the DE wave, it is reasonable to assume that the $D_{\perp}$ is equal to 1 , since the wavelength of the DE mode coupled with the excitation light is about $500 \mathrm{~nm}$ which are much larger than the film thickness of about $20 \mathrm{~nm}$. Therefore, the energy of the DE 
mode can be expressed, as follows:

$$
\begin{aligned}
(\omega / \gamma)^{2}=\mathrm{H}(\mathrm{H}+4 & \left.\pi \mathrm{M}_{\mathrm{s}}-2 \mathrm{~K}_{\mathrm{u}} / \mathrm{M}_{\mathrm{s}}\right) \\
& +\left(2 \pi \mathrm{M}_{\mathrm{s}}-\mathrm{K}_{\mathrm{u}} / \mathrm{M}_{\mathrm{s}}\right)^{2}[1-\exp (-2 \mathrm{kS})],-- \text { (3) }
\end{aligned}
$$

where $\omega$ is a frequency of the spin-wave. $\gamma$ is the spectroscopic splitting factor,

$$
\gamma=\gamma_{\mathrm{e}} \mathrm{g} / 2,--(4)
$$

where $\gamma_{\mathrm{e}}$ is the spectroscopic splitting factor for free electrons and $\mathrm{g}$ is the gyro-magnetic ratio. $\mathrm{H}$ is an external magnetic field parallel to the film plane. $\mathrm{k}$ is a wavenumber of the surface spin-wave contributing the Brillouin scattering,

$$
\mathrm{k}=2 \sin \theta \quad(2 \pi / \lambda),--(5)
$$

where $\theta$ is an angle between the wave vector of an incident light and a film normal, and $\lambda$ is a wavelength of the excitation light. $S$ is a thickness of the magnetic film. Therefore, once the $M_{s}$ value is obtained by VSM, it is possible to calculate the $\mathrm{K}_{\mathrm{u}}$ as well as the $\mathrm{g}$-value from the energy dependence of the DE mode on the external magnetic field.

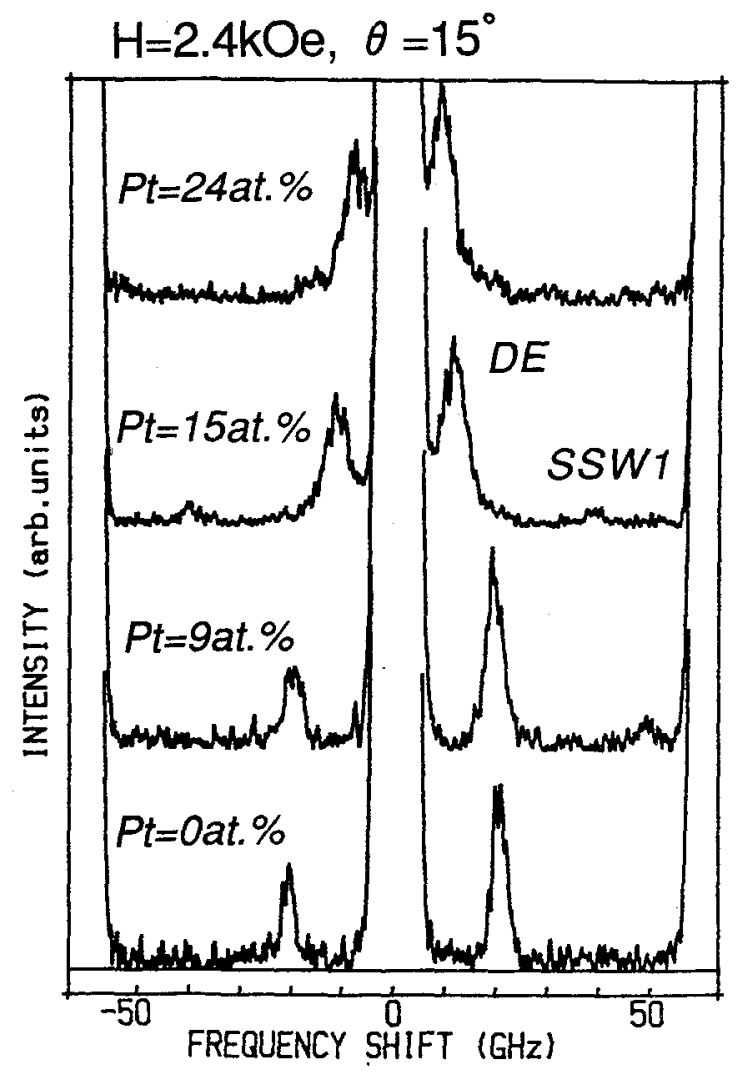

Fig.3 Spin-wave Brillouin spectra in Type-II CoPt alloy films at various $P t$ contents, under magnetic field of $2.4 \mathrm{kOe}$ parallel to the film plane.

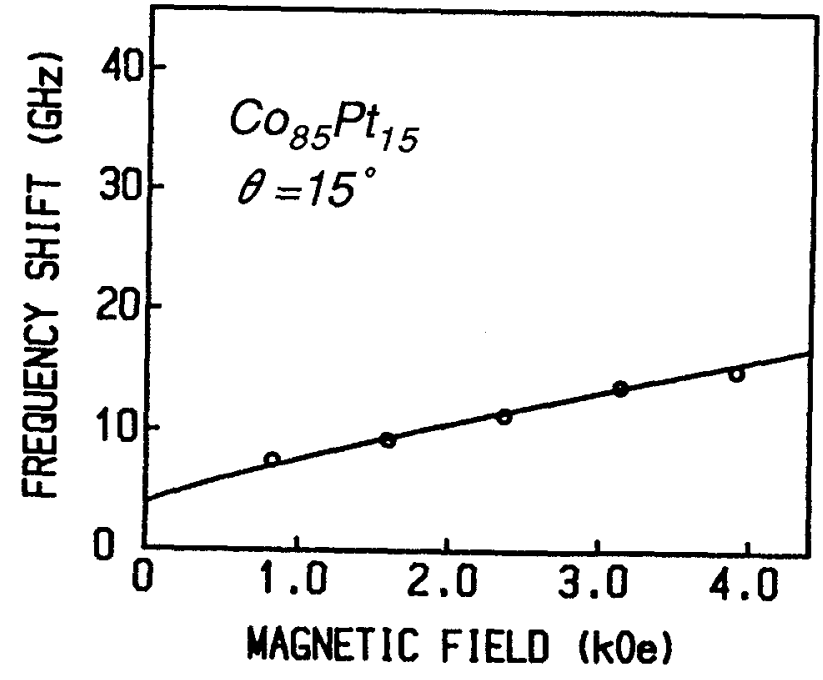

Fig.4 Magnetic field dependence of the DE wave in the Type II-Co ${ }_{85} \mathrm{Pt}_{15}$ film.

Figure 4 shows the frequency shift of the DE wave in the Type II $-\mathrm{Co}_{85} \mathrm{Pt}_{15}$ film as a function of the extemal magnetic field. A solid line is drawn by a best-fit calculation using eqs. (3) $\sim(5)$ and magnetic parameters listed in Table I. It should be noted that magnetic constants derived here were obtained from the magnetic field affecting the spin-wave with a wavelength of submicron which was much larger than the grain size. The $\mathrm{g}$-values in the Type II films with the Pt contents of 15 and 24at.\%, which show the strong perpendicular anisotropy, are significantly smaller than those in the Type I - $\mathrm{Co}_{86} \mathrm{Pt}_{14}$ film. This result is similar to that obtained in $\mathrm{Fe}$ superlattices with large uniaxial anisotropies perpendicular to the film plane[4]. It has been pointed out that the magnetic damping expressed by the Gilbert damping factor can reduce the g-value[5]. Microscopic origin of this effect and the relation to the magnetic anisotropy are subjects to be investigated.

Finally, the effect of a c-axis orientation on the width of the spin-wave spectra is considered. The width of the DE peak depends both on the Pt content and on the film type, as shown in Table I. It has been reported that the spin-wave spectra are markedly broadened with increasing $\mathrm{Pt}$ content up to $15 \mathrm{at} . \%$, in CoPt alloy films with the random orientation of the hcp lattice[6]. The width of that spin-wave spectra correlates well with the in-plane coercivity. Those behaviors are explained by the magnetic 
inhomogeneity due to the grain growth with a three dimensional random orientation of the crystalline magnetic anisotropy. In this experiment, the peak width in the Type II film is observed to be much narrower than that in the Type I. This narrowing of the width of the DE peak in the Type II films is qualitatively interpreted by the decrease in the degree of the three-dimensional dispersion of the crystalline anisotropy due to the perpendicular c-axis orientation of the hcp lattices. Narrower peaks in the films having a perpendicular anisotropy indicates that the collective behavior of spins is more uniform in the size of a wavelength of the DE-mode. Therefore, it is expected that the collective ordering of the spins coupled with the exchange and magnetostatic interactions is more uniform in the films having a perpendicular anisotropy, in the highdensity magnetic recording, since the length of the recording bit becomes less than $1 \mu \mathrm{m}$ in such high-density recording.

\section{CONCLUSION}

We have studied Damon-Eshbach type surface spin-waves in CoPt alloy films with the perpendicular caxis orientation of hcp lattices to the film plane, and hence with the uniaxial perpendicular magnetic anisotropy, by changing the Pt content up to 24 at.\%. The spin-wave energy in such type of the films is remarkably affected by the perpendicular magnetic anisotropy. From the dependence of the spin-wave energy on the magnetic field, the g-value and uniaxial anisotropy energy are derived. The effect of the c-axis dispersion on the width of the DE mode is also discussed.

\section{【REFERENCES】}

[1]Y.Shiroishi, Y.Matsuda, K.Yoshida, H.Suzuki, T.Ohno, N.Tsumita, M.Ohura and M.Hayashi, IEEE Trans. Magn., MAG-24, 2730(1988).

[2]J.R.Sandercock, in Light Scattering in Solids III, edited by M.Cardona and G.Guntherrodt, (Springer, Berlin, 1982), p173.

[3]R.W.Damon and J.R.Eshbach, J.Phys.Chem.Solid, 19, 308(1961).

[4]J.R.Dutcher, B.Heinrich, J.F.Cochran, D.A.Steigerwald and W.F.Egelhoff Jr., J.Appl.Phys., 63, 3464(1988).

[5]B.Heinrich, K.B.Urquhart, A.S.Arrott, J.F.Cochran, K.Myrtle, and S.T.Purcell, Phys.Rev.Lett., 59, 1756(1987).

[6]A.Murayama, M.Miyamura, K.Nishiyama, K.Miyata and Y.Oka, J.Appl.Phys., 69, 5661(1991).

Table I Magnetic parameters in CoPt alloy films.

\begin{tabular}{lllll} 
Alloy & Type I $-\mathrm{Co}_{86} \mathrm{Pt}_{14}$ & Type II $-\mathrm{Co}_{91} \mathrm{Pt}_{9}$ & $\mathrm{II}-\mathrm{Co}_{85} \mathrm{Pt}_{15}$ & $\mathrm{II}-\mathrm{Co}_{76} \mathrm{Pt}_{24}$ \\
\hline Thickness $[\AA]$ & 250 & 230 & 240 & 200 \\
In-plane Coercivity* [Oe] & 670 & 166 & 569 & 324 \\
In-plane Squareness* & 0.87 & 0.85 & 0.56 & 0.63 \\
$\mathrm{M}_{\mathrm{s}}{ }^{*}$ [gauss] & 1060 & 870 & 810 & 750 \\
g-value** $^{* *}$ & 2.0 & 2.1 & 1.7 & 1.7 \\
$\mathrm{~K}_{\mathrm{u}}{ }^{* *}[\mathrm{erg} / \mathrm{cc}]$ & $<1.1 \times 10^{4}$ & $1.7 \times 10^{4}$ & $1.5 \times 10^{6}$ & $8.5 \times 10^{5}$ \\
DE mode-FWHM ${ }^{* *}[\mathrm{GHz}]$ & 9.9 & 3.5 & 5.4 & 4.6 \\
\hline
\end{tabular}

* obtained by VSM, ** obtained by Brillouin light scattering, 